\title{
Protective role of sardine protein (Sardina pilchardus) against high fructose-induced metabolic syndrome rat
}

\author{
Z. Madani ${ }^{1}$, D. Ait Yahia ${ }^{1}$, F. Boukortt ${ }^{1}$, K. Louchami ${ }^{2}$, A. Sener ${ }^{2}$ and M. Bouchenak ${ }^{1}$ \\ ${ }^{1}$ Laboratoire de Nutrition Clinique et Métabolique, Faculté des Sciences, Université d'Oran Es-Sénia, 31000 Algeria and \\ ${ }^{2}$ Laboratoire d'Hormonologie Expérimentale, Bât GE-CP 626, Route de Lennik 808, B-1070 Bruxelles, Belgique
}

Feeding rats high fructose diet affects both glucose and lipid metabolism which result in a cluster of metabolic abnormalities such as glucose intolerance, hypertension, dyslipidaemia and reduced insulin action ${ }^{(1,2)}$.

The aim of the present study was to evaluate the potential influence of sardine protein on biomarkers of oxidative stress and inflammation in high fructose diet-induced metabolic syndrome rat.

Male Wistar rats $(n=26)$ weighing $200 \pm 0 \mathrm{~g}$ were divided to four groups of six rats each and fed $20 \%$ casein (Cas) or sardine protein (SP) with or without $64 \%$ fructose (F) for 2 months. Plasma glucose, insulin, lipids, tumour necrosis factor- $\alpha$ and leptin levels were determined. Lipid peroxidation and hydroperoxydes, vitamin (Vit) E and Vit C and antioxidant enzymes activities in liver homogenates were assayed.

The SPF diet diminished by $21 \%$ and $51 \%$ plasma glucose and glucose intolerance as compared to CasF $(P<0.05)$. Plasma glucose was increased by $39 \%$ and $28 \%$ in CasF and SPF as compared to Cas and SP, respectively. Plasma insulin levels were reduced by $35 \%$ in SPF $v$. CasF and by $38 \%$ in SP $v$. Cas but were increased by $31 \%$ in SPF $v$. SP. HOMA-IR index was 1.72- and 1.91-fold lower in SPF $v$. CasF and in SP $v$. Cas, respectively. Moreover, HOMA index was enhanced by $47 \%$ in CasF $v$. Cas and $64 \%$ in SPF $v$. SP. Plasma cholesterol, triglycerides and free fatty acids levels were significantly lower in SPF and SP rats compared to CasF and Cas. Consumption of SP diet with or without fructose lowered significantly plasma TNF- $\alpha$ values as compared with casein. The high fructose in both the protein diets increased plasma TNF- $\alpha$ by $98 \%$ in CasF $v$. Cas and by $143 \%$ in SPF $v$. SP. Plasma leptin was lower 1.23-fold in SPF than CasF and 2.40-fold in CasF than Cas and 2.28-fold in SPF than SP. Liver hydroperoxides concentrations were enhanced by $18 \%$ and $11 \%$ in CasF $v$. Cas groups and in SPF $v$. SP groups, respectively. Lowered values were noted in liver superoxide dismutase activity in CasF $v$. Cas $(38 \%)$ and in SPF $v$. SP (34\%). Glutathione peroxidase activity was 1.6- and 1.42-fold lower in CasF v. Cas and in SPF v. SP, respectively. No significant difference was observed in catalase activity. Feeding SP diet with or without fructose increased significantly liver Vit C. Hepatic Vit C contents were reduced by $39 \%$ in CasF $v$. Cas and $33 \%$ in SPF $v$. SP. Liver Vit E was significantly higher in SP v. Cas. In addition, Vit E levels were 1.53- and 1.63-fold lower in CasF v. Cas and in SPF v. SP rats.

In conclusion, sardine proteins have hypoglycaemic, hypoinsulinaemic and hypolipidaemic effects and improve oxidative and inflammatory stress induced by a high-fructose diet, suggesting that these proteins may have a beneficial action on functional characteristics of pancreatic beta cells, probably by improving insulin and thereby insulin resistance prevention and may contribute to the low risk of coronary heart disease in patients with the metabolic syndrome.

1. Cussons AJ, Stukey BG \& Watts GF (2007) Curr Diab Rep 7, 66-73.

2. Faeh D, Minehira K, Schwarz JM et al. (2005) Diabetes 54, 1907-1913. 